\title{
Correction to: The Heterogeneity of Cancer Metabolism
}

\author{
Anne Le
}

\section{Correction to:}

A. Le (ed.), The Heterogeneity of Cancer Metabolism, Advances in Experimental Medicine and Biology 1311 https://doi.org/10.1007/978-3-030-65768-0

After initial publication of the book, various errors were identified that needed correction. All corrections listed below have been updated within the current version.

\section{Chapter 1:}

1. Figure 2 was missing the icons for colon and pancreas. The correct figure has been updated.

\section{Chapter 6:}

1. P.92: In the $2^{\text {nd }}$ paragraph of section 2 , the word "is" has been added between the words "fibroblasts" and "associated" (that is, "fibroblasts associated" to "fibroblasts is associated").

2. P.98: The tumor image in figure 3 was having white background. This figure has been updated to have the tumor match with the background color.

\section{Chapter 7:}

1. P.108: In section 3.3, the word "redox-homeostasis" has been updated to "redox homeostasis" (that is, "redox-homeostasis and biosynthesis." to "redox homeostasis and biosynthesis.")

\section{Chapter 12:}

1. P.170: In the $2^{\text {nd }}$ paragraph of the Conclusion section, a comma has been added to the sentence (that is, "Thus CSCs" to "Thus, CSCs").

The updated online version of these chapters can be found at: https://doi.org/10.1007/978-3-030-65768-0_1 https://doi.org/10.1007/978-3-030-65768-0_6 https://doi.org/10.1007/978-3-030-65768-0_7 https://doi.org/10.1007/978-3-030-65768-0_12 https://doi.org/10.1007/978-3-030-65768-0_17 https://doi.org/10.1007/978-3-030-65768-0_18 


\section{Chapter 17:}

1. P.230: In the Introduction section, the word "metabolisms" has been changed to "metabolism" (that is, "including metabolisms of glutamine" to "“"including metabolism of glutamine").

\section{Chapter 18:}

1. P.253: In section 2.3, in the sentence "The inhibition of GLS1 expression has..." the word "researches" has been updated to "research". 